\title{
A New Digital Sense Multiple Access (DSMA) Protocol for High-Speed Wireless Networks*
}

\author{
Kin K. Leung, ${ }^{1}$ Jin-Meng $\mathrm{Ho}^{2}$ and Herman $\mathrm{Chien}^{3}$ \\ ${ }^{1}$ AT\&T Labs, Room 4-120, 100 Schulz Drive, Red Bank, NJ 07701 \\ 2 AT\&T Labs, Room 15B-050, 67 Whippany Road, Whippany, NJ 07981 \\ ${ }^{3}$ AT\&T Wireless Services, 14520 NE 87th Street, Redmond, WA 98052
}

\begin{abstract}
In this paper, we propose a new Digital Sense Multiple Access with Delayed Transmission (DSMA/DT) protocol for reverse channel in high-speed wireless networks. The new protocol is motivated by the observation that the existing DSMA protocol does not yield satisfactory throughput for long round-trip propagation and processing delay, which occurs in outdoor, high-speed environments or when the receiver hardware requires long signal processing time. The new DSMA/DT protocol is intended to reduce the performance impacts of the round-trip delay. Several optional features: look-ahead busy/idle flag, seizure queueing and reserved time slots are also devised for the new protocol. While requiring at most two additional status bits on the forward channel and no additional hardware capability, these features further enhance the protocol performance, and enable constant-bit-rate service with little added complexity in control.

The channel throughput of the DSMA/DT protocol and the optional features are analyzed. Closed-form expressions for the throughput are obtained. Our numerical results show a significant improvement of throughput for the new protocol compared to the existing DSMA protocol for nonnegligible round-trip delay relative to packet transmission time. For the superior performance and simplicity, the DSMA/DT protocol will be appropriate for use in high-speed wireless networks.
\end{abstract}

Subject Area: Multiple Access Methods

* Please forward correspondence to Kin K. Leung at his address, or by email kkleung@research.att.com, phone (732)345-3153 and fax (732)345-3038. 


\section{INTRODUCTION}

Much research efforts have been spent on the subject of multiple access control (MAC) protocols in communications networks since late 1960's; see text [RS90], [BG93] and [T96], and recent survey papers [P95] and [SK97b]. Among many MAC protocols reported in public literature, the Carrier Sense Multiple Access with Collision Detection (CSMA/CD) protocol is widely used in wired local area networks because of its simplicity and high throughput performance. However, due to radio capturing effects, a user in wireless networks cannot transmit and listen on the same radio channel simultaneously, as required by the CSMA/CD protocol. To overcome this difficulty, the Digital Sense Multiple Access (DSMA) protocol transmits channel and decode status flags on the forward channel (i.e., from base station to mobile unit) to indicate the status of the associated reverse channel. The channel and decode flags are used to signify whether the reverse channel is busy or idle and whether the data block that was just received on the reverse channel has been decoded without any errors or not, respectively. With the status information about the reverse channel, the DSMA protocol operates in a way similar to the slotted CSMA/CD protocol. The DSMA protocol has been used in Cellular Digital Packet Data (CDPD) networks [SK96, SK97a] to support narrowband data services. (See [PL94] for an extensive survey of issues pertinent to wireless data communications.)

As technology advances and customers' demand for broadband, multimedia services grow, broadband wireless networks such as wireless ATM have become a hot topic of research. As various types of traffic (e.g., voice, data and video) have different performance requirements such as bandwidth guarantee, packet loss probability, and delay and its jitter, many new MAC protocols have been proposed for the high-speed, packet wireless networks. For example, using the concept of reservation ALOHA [CRWOH73] originally proposed for satellite communications, the Packet Reservation Multiple Access (PRMA) protocol was presented in [GVGB89] and [NGT91] for voice communications in indoor wireless environments. The Resource Auction Multiple Access (RAMA) in [A93] uses an auction process, instead of the slotted ALOHA contention, to assign radio resources for improving throughput. By observing the shortcomings of the PRMA and RAMA protocols, the Dynamic Reservation Multiple Access (DRMA) [QL96a] and the Dynamic Reservation Multiple Access with Piggybacked Reservation (DTDMA/PR) [QL96b] were proposed for packet wireless networks with multimedia traffic. By a combined use of a polling mechanism and minislots for reservation, the Centralized Packet Reservation Multiple Access (C-PRMA) [BBFMZ97] further 
improves upon the PRMA protocol for local wireless networks with microcells. Using slotted ALOHA for mini-reservation packets, a multiservice dynamic reservation TDMA protocol was proposed in [WGJR93] and [RW94] for wireless ATM networks with constant-bit-rate (CBR) and variable-bit-rate (VBR) services.

We observe that either explicitly or implicitly, the MAC protocols for broadband wireless networks referenced above assume that signal propagation plus processing delay is negligibly small relative to the packet transmission time. This assumption is valid for wireless local-area networks, microcell environments or networks with low data rate. When it is invalid in other scenarios, the protocol throughput performance will not be satisfactory.

To illustrate this point further, let us consider an outdoor cellular network with a data rate of 2 Mbps and a cell radius of several kilometers. The shortest packet length for the reverse channel at the MAC layer can be an TCP/IP acknowledgement, which is about 50 bytes. In this case, the packet transmission time is $200 \mu \mathrm{s}$. Even assuming that signal propagates at the speed of light, the round-trip propagation and processing delay can easily reach $100 \mu \mathrm{s}$, which is not negligible relative to the packet transmission time. Further increasing the data rate will make the situation even worse. Of course, packet length is often longer than 50 bytes in many applications. However, in the popular web browsing application, it is not uncommon to find that a good portion of traffic on the reverse channel is TCP/IP acknowledgements, while pages and pages of information are downloaded to users on the forward channel. The relatively long propagation plus processing delay makes many of the MAC protocols inefficient. Consequently, these protocols with the assumption of short propagation plus processing delay will become inapplicable to the outdoor, high-speed environment. In particular, the DSMA protocol will not perform well when the delay is non-negligible when compared to packet transmission time, as expected in the high-speed networks.

Certainly, MAC protocols that are insensitive to the propagation and processing delay have been proposed in the literature. For one example, the reservation ALOHA scheme [CRWOH73] was devised for satellite systems with long round-trip delay, although its shortcomings such as unsatisfactory performance in case of heavy traffic and dedication of fixed bandwidth for reservation have been pointed out by [QL96a]. Another example is the Distributed-Queueing Request Update Multiple Access (DQRUMA) [KLE95] and [LKZE96] for wireless packet networks where performance impacts due to propagation and processing delay can be avoided by appropriately 
adjusting the timing between the forward and reverse channel. As discussed below, a potential shortcoming for the DQRUMA protocol in high-speed wireless networks is its hardware complexity.

Among many techniques, a common way to improve performance of MAC protocols such as those in [A93], [QL96a], [QL96b], [KLE95] and [BBFMZ97], is to use mini-slots for reservation and long slots for information data. Indeed, such use of mini-slots can improve performance at the link layer. However, its overhead and complexity introduced to the physical layer is seldom considered. Specifically, regardless of the actual length of a slot, special symbols (i.e., bit patterns) must be transmitted at the beginning of each slot for channel synchronization and equalization. The number of these symbols required for these purposes depends on the delay spread and other characteristics of the radio link. So, the performance improvement of the mini-slots is reduced when considering such physical-layer overhead. More importantly, mixed slot sizes complicate the hardware design for the radio receiver. It is so because the receiver hardware often employs a pipeline architecture, which is incompatible with variable slot lengths. One natural approach to handling the situation is to design the digital-signal-processing hardware fast enough to process signal arriving in sequence of minislots, without incurring much processing delay. Such hardware complexity will become a significant challenge, especially for high-speed radio networks. This hardware consideration has led us to focus constant slot size. Combining this with the simplicity of the DSMA protocol have motivated us to enhance it for broadband wireless networks in this paper.

The rest of this paper is organized as follows. Section 2 introduces a new DSMA with Delayed Transmission (DSMA/DT) protocol to circumvent the throughput degradation due to long propagation and processing delay. In Section 3 to 5, we introduce three optional features: look-ahead busy/idle bit, seizure queueing and time-slot reservation, to further enhance the performance and flexibility of the DSMA/DT protocol. Section 6 analyzes the channel throughput for the DSMA/DT protocol with and without these features, while Section 7 presents several numerical examples to illustrate the merits of the new protocol. Finally, we have our conclusions in Section 8.

\section{THE DSMA WITH DELAYED TRANSMISSION (DSMA/DT) PROTOCOL}

Consider a wireless network where transmission on the forward and reverse channel is slotted and user terminals are synchronized with their base station. For brevity, let us refer to the round-trip signal propagation plus processing delay as the round-trip delay (denoted by $T$ ). In the context of 
DSMA protocol, $T$ is the number of time slots counting from a time slot in which data is sent on the reverse channel by a user to the earliest time slot by which the busy/idle (B) and decode (D) flags associated with the former slot have been received and processed from the forward channel. In the CDPD networks [SK97a], the B and D flags incur different round-trip delays because of their different processing requirements as the B flag is determined only by the detection of signal, while the $\mathrm{D}$ flag requires data detection and error checking. We assume that the B and D flags incur identical delay here. This assumption is appropriate for: a) the high-speed, outdoor environment as the propagation delay is a major component of the round-trip delay, and b) harsh radio environments where the flags have to be protected by error control codes, thus reducing the difference between their processing requirements.

When the round-trip delay is long, the obsolete channel busy/idle and decode status information on the forward channel cause two impacts on the DSMA protocol performance: 1) high collision probability as other terminals are not aware of ongoing transmission on the reverse channel promptly, thus lowering throughput, and 2) long delay in stopping ongoing transmission in case of collision, thus wasting bandwidth needlessly. The DSMA with Delayed Transmission (DSMA/DT) protocol is devised to overcome these shortcomings, while preserving the simplicity of the DSMA protocol.

The key idea for the DSMA/DT protocol to reduce the impacts of long round-trip delay is that users do not send a whole packet when sensing the channel idle. For this reason, in the new protocol, packets are divided into multiple data blocks where the block size is chosen to be the smallest but efficient for processing by the receiver hardware for a given error-control code in use (e.g., ReedSolomon code). The channel time is slotted such that a time slot can carry one data block. The channel status for each slot on the reverse channel is indicated by the B and D flags in a corresponding time slot on the forward channel in the DSMA/DT protocol. That is, $B=1$ if data signal is received and 0 otherwise; $D=1$ if the data block is successfully decoded and 0 otherwise.

The DSMA/DT protocol requires that each user with packets for transmission first sense the reverse channel by observing the $\mathrm{B}$ flag prior to transmission. Upon finding the channel idle $(\mathrm{B}=0)$, a ready user transmits one data block in the next time slot on the reverse channel. Once deciding to transmit, the user observes the D flag for the following $T$ time slots (indexed by 1 to $T$ ) received on the forward channel. If (1) the D flag for all time slot 1 to $T-1$ does not indicate any successful decode (reception) and (2) the D flag for time slot $T$ shows that the first data block is successfully 
decoded, then the channel is considered to be seized by the user who can send the rest of the packet on the reverse channel, starting from time slot $T+1$. To ensure no collision with the latter transmission, upon successful decoding of the very first data block received from any user, the base station artificially sets the B flag to 1 in the next $T$ time slots. (That is, the first $\mathrm{B}=1$ flag is associated with the time slot on the reverse channel at which the first successful data block is sent.) If condition (1) holds but (2) does not, the user is probably involved in a transmission collision with others, and will back off (e.g., according to an exponential back-off procedure) before attempting retransmission. If the D flag for any of time slot 1 to $T-1$ indicates a successful decode, then the channel has already been seized and the user defers its transmission with a random delay. Since the new protocol requires a user to delay possible transmission of remaining data blocks until the associated D flag signifies the channel seizure for the user, the protocol is thus called the DSMA with Delayed Transmission (DSMA/DT) protocol. Further, the transmission of remaining data blocks is referred to as the delayed transmission. As to be discussed later, several optional features can be adopted to further enhance the DSMA/DT performance and provide additional flexibility to meet different needs.

Let us examine why the DSMA/DT protocol operates as expected. As illustrated in Figure 1, there are three possible cases. In case (a), both condition (1) and (2) hold and they signify the channel seizure only by user 1 . This fact is reflected by the sequence of D flags, and becomes known to the user and all other users after the round-trip delay. Combining this with the $B=1$ flag in the first $T$ slots following the successful decode of the first data block, no other user will collide with the initial $T$ blocks of the delayed transmission by user 1 . Since the B flag is continued to be set to 1 as long as signal is received beyond the $T$ slots, user 1 will not experience any collision in the entire packet transmission. For case (b) in the figure, transmission collision is indicated by unsuccessful decode flag $D=0$ after the round-trip delay. The users involved in the collision go through a back-off procedure. In case (c), user 1 upon finding $\mathrm{B}=0$ (channel idle) sends his first data block, without realizing that user 2 has sent earlier. This scenario is possible because the retarded B and D flags enable multiple users without knowing the current channel status to send simultaneously within the "collision window" of round-trip delay. Since each user continues to monitor the D flag for $T$ slots after sending his first block, $D=1$ in any slot from 1 to $T-1$ reveals to user 1 that a data block successfully received by the base station is sent earlier by user 2 . As a result, user 1 defers his transmission by a random delay before re-attempt, while user 2 seizures the channel and transmits the rest of his data blocks as shown in the figure. 
To an extent, the DSMA/DT protocol can be viewed as a reservation protocol where the first data block serves as an implicit "reservation" request and the remaining data is transmitted after a fixed delay ( $T$ time slots) following the success of reservation. Similar to the CSMA protocol, one advantage of the new protocol is the use of channel sensing as a means to reduce collision in the "reservation" process. In addition, similar to the PRMA [GVGB89] and the DRMA [QL96a], the DSMA/DT protocol has an advantage of not dedicating a fixed amount of bandwidth for the "reservation." Rather, it is dynamically changed according to contention situations. For example, frequent collision at high traffic load results into many time slots used by users for sending their first data block, thus providing efficient sharing of bandwidth between "reservation" process and contention-free transmission. As discussed earlier, another important advantage of the DSMA/DT protocol is its fixed time-slot size, which can simplify the receiver hardware design.

It is worth noting that the D flag in the DSMA/DT protocol serves two purposes. First, as in the DSMA protocol, the flag reflects decoding result. Second, the D flag in the new protocol also helps users recognize when the channel seizure occurs. Although the DSMA/DT protocol guarantees no collision after seizure, due to radio impairments, it is possible to receive a $D=0$ flag for the transmission of remaining data blocks. When such happens, the user aborts immediately and repeats the transmission at a later time. We point out that such dual use of the D flag will become insufficient when the DSMA/DT protocol adopts the feature of seizure queueing, which is discussed later.

We also remark that the DSMA/DT protocol can operate properly with the D flag alone (i.e., without the B flag) plus the following changes. First, each user senses the channel by examining the D flag; that is, $\mathrm{D}=0$ and 1 indicates channel idle and busy, respectively. As before, base station sets the D flag according to the decode result. In addition, base station artificially sets the D flag (instead of the B flag) to 1 for $T$ time slots following the successful decode of the first data block. The rest of the protocol remains unchanged. We choose to present the protocol using both B and D flag because they are needed in several optional features to further enhance the protocol performance or offer additional flexibility to provide constant-bit-rate service. These features are the subject of the following sections. 


\section{LOOK-AHEAD BUSY/IDLE FLAG}

Upon examination, the DSMA/DT protocol still suffers from the long round-trip delay. Specifically, the B flag takes $T$ time slots to reset to zero after the completion of a successful transmission; see an example in Figure 2a with $T=3$. The delay wastes bandwidth because no user will attempt to transmit until the channel is "sensed" idle (i.e., the B flag turns zero). One method to avoid such waste is to include a length indicator bit (denoted by $I$ ) in each data block. As shown in Figure $2 \mathrm{~b}$, the user sets $I$ to 1 if the remaining data of a packet require more than $T$ time slots to transmit, 0 otherwise. As a result, if no signal is received in a slot on the reverse channel, its associated B flag is set to 0 on the forward channel. Otherwise, the B flag is set equal to the I bit in the data block just received. In case the received signal cannot be decoded successfully, the B flag is set to 1 to avoid potential collision. This feature is called Look-Ahead Busy/Idle Flag. Note that this look-ahead method of setting the B flag does not apply to the next $T$ time slots immediately following a success decode of the first data block as the flag is always set to 1 to avoid collision in the beginning of the delayed transmission according to the DSMA/DT protocol. It can be seen readily that if a packet consists of at least $T+1$ data blocks, the look-ahead flag can fully eliminate the bandwidth waste due to the round-trip delay, following a successful packet transmission.

An alternative approach to the use of length indicator bit is to encode the packet length in terms of the number of slots in the first data block. Based on the packet length, the base station is responsible for setting the $\mathrm{B}$ flag in the corresponding time slots accordingly. This approach may perform better than that of the indicator-bit technique, depending on the packet length variability and the overhead incurred in embedding the I bit and packet length in the data blocks. Nevertheless, we assume that the approach of length indicator bit is used for the look-ahead flag in the rest of this paper.

\section{SEIZURE QUEUEING}

In the DSMA/DT protocol, it is possible that the first data block of a user can be successfully received, although the channel has been seized by another user. For example, see case (c) in Figure 1. Accordingly, the former user has to defer or back off with his first data block subject to retransmission. This results into a waste of bandwidth, thus motivating us to devise the feature of seizure queueing (SQ). The main idea of this feature is to let users having their first data block 
successfully received (i.e., those who seize the channel) queue up and transmit their remaining data blocks in the order of their channel seizure.

Let us refer to the DSMA/DT protocol with the look-ahead busy/idle flag and the seizure queueing as the DSMA/SQ protocol. In the enhanced protocol, three flags are needed: the B, D, and E flags on the forward channel to reflect the status of each time slot on the reverse channel. As before, the B and D flag indicate channel busy/idle and decode success/failure, respectively, while the E flag is introduced to signify the ending of a packet transmission from a user when $E=1$.

The DSMA/SQ protocol operates as follows. As for the DSMA/DT protocol, each packet is divided into multiple data blocks. A ready user first senses the channel. If $B=1$, the user defers his transmission after a random delay. Otherwise $(B=0)$, he transmits his first data block, and resets a local variable $Q$ to zero, which shows his queueing position while waiting for possible transmission of other data blocks of the packet. Then, the user updates his queueing position by setting $Q=Q+D$ for the D flag received from the forward channel for each of the next $T-1$ time slots. This is appropriate because a $D=1$ flag during any of these slots reveals that another user has his first data block received successfully (i.e., the user seizes the channel). By the same principle, if the D flag for time slot $T$ equals 1 , this indicates a successful decode of the first data block from the user and there are $Q$ other users ahead of the former user for transmission of remaining data blocks. Otherwise, the user is involved in collision and subject to a back-off procedure prior to re-attempt.

After the channel seizure by a user, he updates his queueing position by setting $Q=Q-E$ for every E flag received from the forward channel. It is so because $E=1$ indicates the completion of the delayed transmission by a user who is ahead of the former user. When $Q=0$, the user is the first in the seizure queue, and thus should transmit all his remaining data blocks starting in the next time slot on the reverse channel.

The base station maintains a variable $N_{q}$ to keep track of the number of users who have seized the channel and are waiting for their turn to transmit the remaining data blocks. Initially, $N_{q}=0$. The base station increments $N_{q}$ by 1 when a user seizes the channel, and decrements it by 1 when the delayed transmission of a user is completed. As long as $N_{q}>0$, the B flag is set to 1 . When $N_{q}=0$, the B flag is set according to the look-ahead busy/idle flag discussed above. As for the E flag, base station normally sets it to zero unless it has just received for the first time: (1) a data block with the 
$\mathrm{I}=0$ bit or (2) a data block that cannot be decoded successfully from the delayed transmission of a given user. When either condition (1) or (2) occur, the E flag is set to one for the next time slot and immediately reset to zero thereafter. If it is possible that a packet consists of only one data block, there will no delayed transmission associated with that packet. In this case, the E flag can also be set to 1 when the following scenario occurs. That is, when $N_{q} \geq 1$ but no signal is received in a time slot

that is supposedly corresponding to the starting slot of a delayed transmission (because the packet in question has one data block), the base station sets the E flag to 1 for that time slot, and resets it to 0 thereafter. Such $E=1$ flag will ensure other users to update their queueing position properly, despite that the user currently at the head of the seizure queue is sending a packet of one data block, thus requiring no delayed transmission. The setting of the $\mathrm{B}, \mathrm{D}$ and $\mathrm{E}$ flags for an example with $T=3$ is shown in Figure 3.

\section{RESERVED TIME SLOTS FOR CONSTANT-BIT-RATE SERVICE}

As the constant-bit-rate (CBR) service or services requiring guaranteed bandwidth are expected in future networks, it is desirable to enhance our new MAC protocol to support such services. Let us start with the DSMA/DT protocol. A natural approach to guaranteeing bandwidth is to reserve time slots for exclusive access by a user in a periodic manner. We refer to the DSMA/DT protocol with such reserved slots (RS) as the DSMA/RS protocol.

In the DSMA/RS protocol, the reverse channel time is divided into frames, each of which consists of $M$ time slots. When a time slot is reserved for a user, the same time slot in subsequent frames is dedicated for the user until the CBR connection is terminated. Any slot in a frame can be reserved and reserved slots need not be contiguous in time. The use of the B and D flag in the DSMA/RS protocol is identical to that for the DSMA/DT protocol. In addition, the DSMA/RS protocol also requires an $\mathrm{R}$ flag for each slot to indicate whether it has been reserved or not. Specifically, $R=1$ if the corresponding time slot has already been reserved for a user, and $R=0$ otherwise.

Based on the R flag, users and base stations can identify reserved and non-reserved time slots, and treat all non-reserved slots as a logical channel for transmitting data on a contention basis. In light of this view, if users and base station skip all reserved slots for channel sensing, and transmission of status flags and contention data, the DSMA/DT protocol can be readily applicable to 
the logical channel for access control and resolution of collision without any hardware changes. For the same reason, the DSMA/DT protocol with the look-ahead busy flag and/or seizure queueing feature is also applicable to the logical channel, although we focus mainly on the use of DSMA/DT protocol with the look-ahead flag for contention data transmission in the DSMA/RS protocol in this paper.

To establish a CBR connection, a user simply sends a request block in an idle slot (say slot $i$ ) on the non-reserved, logical channel according to the DSMA/DT protocol. If the request is decoded correctly at the base station, the $\mathrm{D}$ and $\mathrm{R}$ flag are set to 1 for the slot $i+T$ on the logical channel to signify that slot is now reserved for the user. Otherwise, the D and R flag are set to zero to reflect the reservation failure. An example setting of the B, D and R flag for the DSMA/RS is given in Figure 4. Users with unsuccessful reservation can re-attempt after a random delay. Using this method to reserve one time slot at a time, a user can obtain more than one reserved slots per frame by repeating the procedure multiple times. Alternatively, if the successfully decoded request sent in slot $i$ also contains the number of reserved slots requested (say $k$ ), the base station can set the D and R flag to 1 for slots indexed by $i+T$ to $i+T+k-1$ on the logical channel to indicate that these $k$ slots are now reserved for the user.

\section{THROUGHPUT ANALYSIS}

In the following, we analyze the throughput performance of the DSMA/DT protocol, the lookahead busy bit and the seizure queueing. As for the standard treatment of MAC protocol, the focus of our analysis lies on transmission collision, and does not account for power capture, interference and thermal noise. In light of the last two factors, our analysis results can be viewed as upper bound results. Lastly, we explain that the throughput for the DSMA/RS protocol is identical to that for the DSMA/DT protocol with appropriate adjustment of packet length. For the sake of completeness and fair performance comparison, we also analyze the channel throughput for the regular DSMA protocol in the Appendix.

\subsection{Throughput of the DSMA/DT Protocol}

The throughput analysis for the DSMA/DT protocol follows the classical approach by use of renewal theory for the CSMA protocols [KT75]. As shown in Figure 5, the DSMA/DT channel 
alternates between collision period and busy period. During a collision period, either the time slots are idle or there are more than one users transmitting. A collision period ends and the next busy period begins when the first data block is successfully decoded. Then the busy period continues until the delayed transmission is completed and followed by the round-trip delay so that the B flag reflects channel idle again; see Figure 5. Let us assume that the aggregated arrival process of new and retransmitted packets is a Poisson process with intensity of $g$ packets/slot. Thus, when only one data block is transmitted in a slot, the block is considered to successfully decoded with a probability of $g e^{-g}$. Let $\bar{C}$ and $\bar{B}$ denote the average length of collision and busy period in number of slots, respectively. Due to the memoryless property of Poisson traffic, we have

$$
\bar{C}=\sum_{i=0}^{\infty} i\left(1-g e^{-g}\right)^{i} g e^{-g}=\frac{1-g e^{-g}}{g e^{-g}} .
$$

Let $L$ and $\bar{L}$ be the packet length (a random variable) and its average in terms of the number of time slots. As depicted in Figure 5, we have

$$
\bar{B}=T+\bar{L}-1+T \text {. }
$$

Note that within a busy period, the average length of successful transmission (denoted by $\bar{U}$ ) is simply $\bar{L}$. Using the expressions for $\bar{C}, \bar{B}$ and $\bar{U}$, the channel throughput $S$ is given by renewal theory as

$$
S=\frac{\bar{U}}{\bar{C}+\bar{B}}=\frac{g e^{-g} \bar{L}}{1+(2 T+\bar{L}-2) g e^{-g}} .
$$

Let us consider the throughput improvement due to the look-ahead busy flag by use of length indicator bit in each data block. Since the DSMA/DT protocol artificially sets the busy/idle flag to 1 for $T$ slots after successful decode of the first data block (i.e., channel seizure), the busy period has a length of $B=T+T$ when $L \leq T$. One the other, when $L \geq T+1$, the round-trip delay for the lookahead flag after the completion of a packet transmission can be fully avoided. That is, $B=T+L-1$. Thus, taking the average by combining both cases yields

$$
\bar{B}=T+\sum_{i=1}^{T} T \alpha_{i}+\sum_{i=T+1}^{\infty}(i-1) \alpha_{i}
$$

where $\alpha_{i}$ is the probability of an arbitrary packet consisting of $i$ data blocks. As before, the average length of useful transmission in a busy period $\bar{U}$ is equal to $\bar{L}$. Using this fact, the channel throughput 
$S$ for the DSMA/DT protocol with the look-ahead busy flag can be obtained by

$$
S=\frac{\bar{L}}{\bar{C}+\bar{B}}
$$

with $\bar{C}$ and $\bar{B}$ given by (1) and (4), respectively. If each packet has at least $T+1$ data blocks (i.e., $L \geq T+1$ ), substituting this into (4) and then (5) yields

$$
S=\frac{g e^{-g} \bar{L}}{1+(T+\bar{L}-2) g e^{-g}} .
$$

Similarly, if $L \leq T$, we have

$$
S=\frac{g e^{-g} \bar{L}}{1+(2 T-1) g e^{-g}} .
$$

\subsection{Throughput of the DSMA/SQ Protocol}

Similar to the DSMA/DT protocol, the protocol with seizure queueing also alternates between collision and busy period. The average length of collision periods in (1) remains valid for the DSMA/SQ protocol. To obtain a new expression for $\bar{B}$, we define $Q_{k}$ as the probability that, following the first successful transmission of the first data block (first channel seizure) by a user in a busy period, there are $k$ successful transmissions of first data block ( $k$ channel seizures) in the next $T-1$ time slots. Obviously, $k \leq T-1$ as each slot has at most one successful transmission of one data block. Using the memoryless property of Poisson traffic, we get

$$
Q_{k}=\left(\begin{array}{c}
T-1 \\
k
\end{array}\right)\left(g e^{-g}\right)^{k}\left(1-g e^{-g}\right)^{T-k-1} .
$$

Similar to the scenario in Figure 5, each busy period in the DSMA/SQ protocol starts with $T$ time slots where the first slot carries the first successful transmission of a data block following the previous collision period. By the same arguments for (4), $\bar{B}$ for the DSMA/SQ protocol (which includes the look-ahead flag) is 


$$
\begin{aligned}
\bar{B} & =T+\left\lceil\mid \sum_{i=1}^{T} T \alpha_{i}+\sum_{i=T+1}^{\infty}(l-1) \alpha_{i}\right\rfloor\left(1+\sum_{k=1}^{T-1} k Q_{k}\right) \\
& =T+\left\lceil\left|\sum_{i=1}^{T} T \alpha_{i}+\sum_{i=T+1}^{\infty}(l-1) \alpha_{i}\right|\left[1+(T-1) g e^{-g}\right]\right.
\end{aligned}
$$

where the last factor in parentheses is the average number of users seize the channel and thus successfully send their packets in a busy period. As the average packet length is $\bar{L}$, the average length of useful transmission in a busy period is

$$
\bar{U}=\bar{L}\left[1+(T-1) g e^{-g}\right]
$$

By renewal theory, the channel throughput is thus given by $S=\frac{\bar{U}}{\bar{C}+\bar{B}}$ where $\bar{C}, \bar{B}$ and $\bar{U}$ are obtained in (1), (9) and (10), respectively. For closed-form expressions, if $L \geq T+1$, (9) becomes

$$
\bar{B}=T+(\bar{L}-1)\left[1+(T-1) g e^{-g}\right] .
$$

Combining (1), (10) and (11), the channel throughput for $L \geq T+1$ is

$$
S=\frac{\bar{L}\left[1+(T-1) g e^{-g}\right] g e^{-g}}{1-g e^{-g}+g e^{-g}\left\{T+(\bar{L}-1)\left[1+(T-1) g e^{-g}\right]\right\}} .
$$

On the other hand, if $L \leq T$, we obtain from (9) that

$$
\bar{B}=T+T\left[1+(T-1) g e^{-g}\right] .
$$

Consequently, the channel throughput for $L \leq T$ is

$$
S=\frac{\bar{L}\left[1+(T-1) g e^{-g}\right] g e^{-g}}{1-g e^{-g}+g e^{-g} T\left[2+(T-1) g e^{-g}\right]} .
$$

\subsection{Throughput of the DSMA/RS Protocol}

Since reserved slots in the DSMA/RS protocol are not involved in collision, transmission in those slots is considered to be successful as far as throughput performance is concerned. Due to the memoryless property of Poisson process for new and re-attempt packet arrivals, by "cutting" and 
"pasting" of reserved time slots in successive frames for a user together, successful transmission in the reserved slots can be viewed as a long packet transmission by the user. Let $\beta_{i}$ be the probability that there are totally $i$ time slots associated with a successful CBR connection, including the slot at which the reservation request is successfully received and the slots reserved in subsequent frames for the user. We assume that the arrival rates for new packets and CBR connections are $\lambda_{p}$ and $\lambda_{c}$, respectively. Since users send and re-send reservation requests and first data block of packets using the same protocol and back-off procedure, they have identical probability of being decoded successfully. Combining this with the view of treating reserved slots as a long packet, the probability for an arbitrary "packet" (a real packet or a CBR connection) length consisting $i$ slots is

$$
P_{i}=\frac{\lambda_{p} \alpha_{i}+\lambda_{c} \beta_{i}}{\lambda_{p}+\lambda_{c}}
$$

The average packet length is

$$
\bar{L}=\sum_{i=1}^{\infty} i P_{i}
$$

Now, using $\bar{L}$ in (16) and replacing the packet length distribution $\alpha_{i}$ 's in (4) by $P_{i}$ 's in (15), the DSMA/RS throughput is then given by (5) to (7). Similarly, using (15) and (16), the DSMA/RS throughput with the seizure queueing can be readily obtained from the corresponding formulas (12) and (14).

\section{NUMERICAL EXAMPLES}

In this section, we compare the throughput of the DSMA/DT protocol with various features to that of the S-Aloha and the standard DSMA protocol. Figure 6 shows the throughput comparison for a case of $L=4$ and $T=2$. A possible scenario for the parameter setting is that the round-trip (propagation plus processing) delay is $100 \mu s$ and each packet (e.g., a TCP/IP acknowledgement) has 50 bytes divided into 4 data blocks and a link speed of 2 Mbps. In this case, as shown in the figure, the DSMA performance is worse than that for the S-Aloha where each slot is sized to transmit the whole packet. The poor DSMA performance is because of the long round-trip delay that makes the busy and decode flags obsolete by the time they are received by users, thus causing much collision and waste of bandwidth in collision "detection." On the contrary, the DSMA/DT protocol yields a higher throughput than the DSMA protocol does, because the former does not allow users to transmit 
the whole packet when sensing idle, thus reducing the performance impacts due to the obsolete busy flag. When the option of the look-ahead flag is adopted, the throughput is further improved as it reduces the waste of bandwidth due to the long round-trip delay in resetting the busy flag after a successful packet transmission. When the feature of seizure queueing is employed in conjunction with the look-ahead busy flag by use of the length-indicator bit, the DSMA/SQ performs even better than others because all successful transmissions of first data blocks from various users are not wasted and instead they transmit the rest of their data blocks in turn according to their order in the seizure queue. Figure 7 compares the throughput performance with longer packets; that is, $L=11$ and $T=2$. As similar to the CSMA protocol, when comparing Figure 6 and 7, the relative improvement of the DSMA/DT protocol over the regular DSMA protocol diminishes as packet length is increased.

\section{CONCLUSIONS}

In this paper, we have proposed a new Digital Sense Multiple Access with Delayed Transmission (DSMA/DT) protocol for reverse channel in high-speed wireless networks. The new protocol is motivated by the observation that the existing DSMA protocol does not yield satisfactory throughput for long round-trip propagation and processing delay, which occurs in outdoor, high-speed wireless networks or when the receiver hardware requires long signal processing time. The new DSMA/DT protocol is devised to reduce the performance impacts of the round-trip delay. Several optional features: look-ahead busy/idle flag, seizure queueing and reserved time slots are also devised for the DSMA/DT protocol. While requiring at most two additional status flags on the forward channel and no additional hardware capability, these features further enhance the performance and flexibility of the new protocol with little added complexity in control.

The channel throughput of the DSMA/DT protocol and the optional features have been analyzed. Closed-form expressions for the throughput are obtained. Our numerical results show a significant improvement of throughput for the new protocol over the existing DSMA protocol for non-negligible round-trip delay relative to packet transmission time. For the superior performance and simplicity, the DSMA/DT protocol thus is recommended for reverse channel in high-speed wireless networks. 


\section{ACKNOWLEDGMENTS}

Thanks are due to Paul Schachter and Lek Ariyavisitakul for their helpful discussion. 


\section{Appendix: Throughput of the Regular DSMA Protocol}

Similar to the CSMA protocol, the DSMA protocol also alterates between idle and busy period where the latter may contain collided or successful transmission plus the following round-trip delay in

propagating the $\mathrm{B}=0$ flag. Let $\bar{I}$ and $\bar{B}$ denote the average length of the idle and busy period. Let us suppose that packet length is fixed to be $L$ slots here. Further, as assumed in the DSMA/DT throughput analysis, the aggregated new and re-attempt packets arrive according to a Poisson process with intensity of $g$ packets/slot. Thus, the average length of an idle period is

$$
\bar{I}=\sum_{i=0}^{\infty} i\left(e^{-g}\right)^{i}\left(1-e^{-g}\right)=\frac{e^{-g}}{1-e^{-g}} .
$$

Now, let us consider various scenarios in a busy period. First is a successful transmission without collision. In the case, the first slot of the period busy must carry a data block from exactly one user, conditioned on the fact that there is at least one arrival to start the busy period, and there is no other transmission in the following $T-1$ slots (i.e., no arrival in the rest of collision window). By the assumption of aggregated Poisson traffic, the probability for such a condition is given by

$$
\frac{g e^{-g}}{1-e^{-g}} e^{-g(T-1)}
$$

In this scenario, the transmission time in slots is equal to the packet length $L$. The second scenario is that there are more than one users transmitting in the first slot of the busy period and that no user transmits in the following $T-1$ slots. Using similar arguments for (A-2), the probability for this to happen is

$$
\left(1-\frac{g e^{-g}}{1-e^{-g}}\right) e^{-g(T-1)}
$$

In this case, the transmission time is $T$ slots because after the round-trip delay $T$, involved users find out about the collision, and stop transmission.

The third scenario corresponds to the case where regardless of the collision situation in the first slot of the busy period, there are at least one arrival in the second slot and no further arrival in the following $T-2$ slots. This is, the last arrival in the busy period occurs in the second slot. The probability of this situation is 


$$
\left(1-e^{-g}\right) e^{-g(T-2)}
$$

and the transmission time lasts for $T+1$ slots.

There are $T-2$ other similar scenarios where the last arrival occurs in the $3 \mathrm{rd}, 4$ th, ..., to $(T-1)$ st slot in the busy period. Following the deviation for (A-4), for a general scenario that the last arrival occurs in the k-th slot in the busy period, the probability of occurrence is

$$
\left(1-e^{-g}\right) e^{-g(T-k)}
$$

and the transmission time lasts for $T+k-1$ slots where $k=1$ to $T-1$. Combing all these scenarios by using (A-2) to (A-5), the average length of a busy period is

$$
\begin{gathered}
\bar{B}=\frac{g e^{-g}}{1-e^{-g}} e^{-g(T-1)} L+\left(1-\frac{g e^{-g}}{1-e^{-g}}\right) e^{-g(T-1)} T \\
+\sum_{k=2}^{T}\left(1-e^{-g}\right) e^{-g(T-k)}(T+k-1)+T
\end{gathered}
$$

where the last term is due to the round-trip delay in propagating the $\mathrm{B}=0$ flag to users. Note that the average useful transmission time $\bar{U}$ is given by the first term in (6). Hence, the channel throughput for the DSMA protocol is

$$
S=\frac{g e^{-g T} L}{\left(1-e^{-g}\right)(\bar{I}+\bar{B})}
$$

where $\bar{I}$ and $\bar{B}$ are obtained in (A-1) and (A-6), respectively. 


\section{REFERENCES}

[A93] N. Amitay, "Distributed Switching and Control with Fast Resource Assignment/Handoff for Personal Communications Systems," IEEE J. on Select. Areas in Commun., Vol.11, 1993, pp.842-849.

[BBFMZ97] G. Bianchi, F. Borgonovo, L. Fratta, L. Musumeci and M. Zorzi, "C-PRMA: A Centralized Packet Reservation Multiple Access for Local Wireless Communications," IEEE Trans. on Vehicular Tech., Vol.46, No.2, May 1997, pp.422-436.

[BG93] D. Bertsekas and R. Gallager, Data Networks, Prentice Hall, 1993

[CRWOH73] W. Crowther, R. Rettberg, D. Walden, S. Ornstein and F. Heart, "A System for Broadcast Communication: Reservation-ALOHA," Proc. 6th Hawaii Int. Conf. Syst. Sci., 1973, pp.596-603.

[GVGB89] D.J. Goodman, R.A. Valenzuela, K.T. Gayliard and B. Bamamurthi, "Packet Reservation Multiple Access for Local Wireless Communications," IEEE Trans. Commun., Vol.COM-37, pp.885-890, Aug. 1989.

[KLE95] M.J. Karol, Z. Liu and K. Eng, "An Efficient Demand-Assignment Multiple Access Protocol for Wireless Packet (ATM) Networks," Wireless Networks, 1995, pp.267-279.

[KT75] L. Kleinrock and F.A. Tobagi, "Packet Switching in Radio Channels: Part I - Carrier Sense Multiple-Access Modes and Their Throughput-Delay Characteristics," IEEE Trans. on Commun., Vol.COM-23, No.12, Dec. 1975, pp.1400-1416.

[L89] W.C.Y. Lee, Mobile Cellular Telecommunications Systems, McGraw-Hill, New York, 1989.

[LKZE96] Z. Liu, M.J. Karol, E. El Zarki, and K.Y. Eng, "Time-Frequency Slicing with DistributedQueueing Request Update Multiple Access (DQRUMA) for Multi-Rate Wireless Packet (ATM) Networks," in Multiaccess, Mobility and Teletraffic for Personal Communications, B. Jabbari, P. Godlewski and X. Lagrange (Ed.), Kluwer Academic Publishers, Boston, MA (1996), pp.293-306.

[NGT91] S. Nanda, D.J. Goodman, and U. Timor, "Performance of PRMA: A Packet Voice Protocol for Cellular Systems," IEEE Trans. on Vehicular Tech., Vol.40, No.3, 1991, pp.584-598. 
[P95] H. Peyravi, "Multiple Access Control (MAC) Protocols for the Mars Regional Network: A Survey and Assessment," Report prepared for NASA, Cleveland, Ohio, Sept. 1995 (http://mars.mcs.kent.edu/ peyravi/contents.html\#Mars).

[PL94] K. Pahlavan and A.H. Levesque, "Wireless Data Communications," Proc. of the IEEE, Vol.82, No.9, Sept. 1994, pp.1398-1430.

[QL96a] X. Qiu and V.O.K. Li, "Dynamic Reservation Multiple Access (DRMA): A New Multiple Access Scheme for Personal Communication System (PCS)," Wireless Network 2 (1996), pp.117-128.

[QL96b] X. Qiu, V.O.K. Li and J. Ju, "A Multiple Access Scheme for Multimedia Traffic in Wireless ATM," Mobile Networks and Applications 1 (1996), pp.259-272.

[RS90] R. Rom and M. Sidi, Multiple Access Protocols, Springer-Verlag, 1990.

[RW94] D. Raychaudhuri and N.D. Wilson, "ATM-Based Transport Architecture for Multiservices Wireless Personal Communication Networks," IEEE J. on Select. Areas in Commun., Vol.12, 1994, pp.1401-1414.

[SK96] M. Sreetharan and R. Kumar, Cellular Digital Packet Data, Artech House, 1996.

[SK97a] D. Saha and S.E. Kay, "Cellular Digital Packet Data Network," IEEE Trans. on Vehicular Tech., Vol.46, No.3, Aug. 1997, pp.697-706.

[SK97b] D. Sobirk and J.M. Karlsson, "A Survey of Wireless ATM MAC Protocols," presented at International Conference of Performance and Management of Complex Communication Networks, Nov. 17-21, 1997, Tsukuba, Japan.

[T96] A.S. Tanenbaum, Computer Networks, Third Edition, Prentice-Hall, 1996.

[WGJR93] N.D. Wilson, R. Ganesh, K. Joseph and D. Raychaudhuri, "Packet CDMA Versus Dynamic TDMA for Multiple Access in an Integrated Voice/Data PCN," IEEE J. on Select. Areas in Commun., Vol.11, 1993, pp.870-884. 

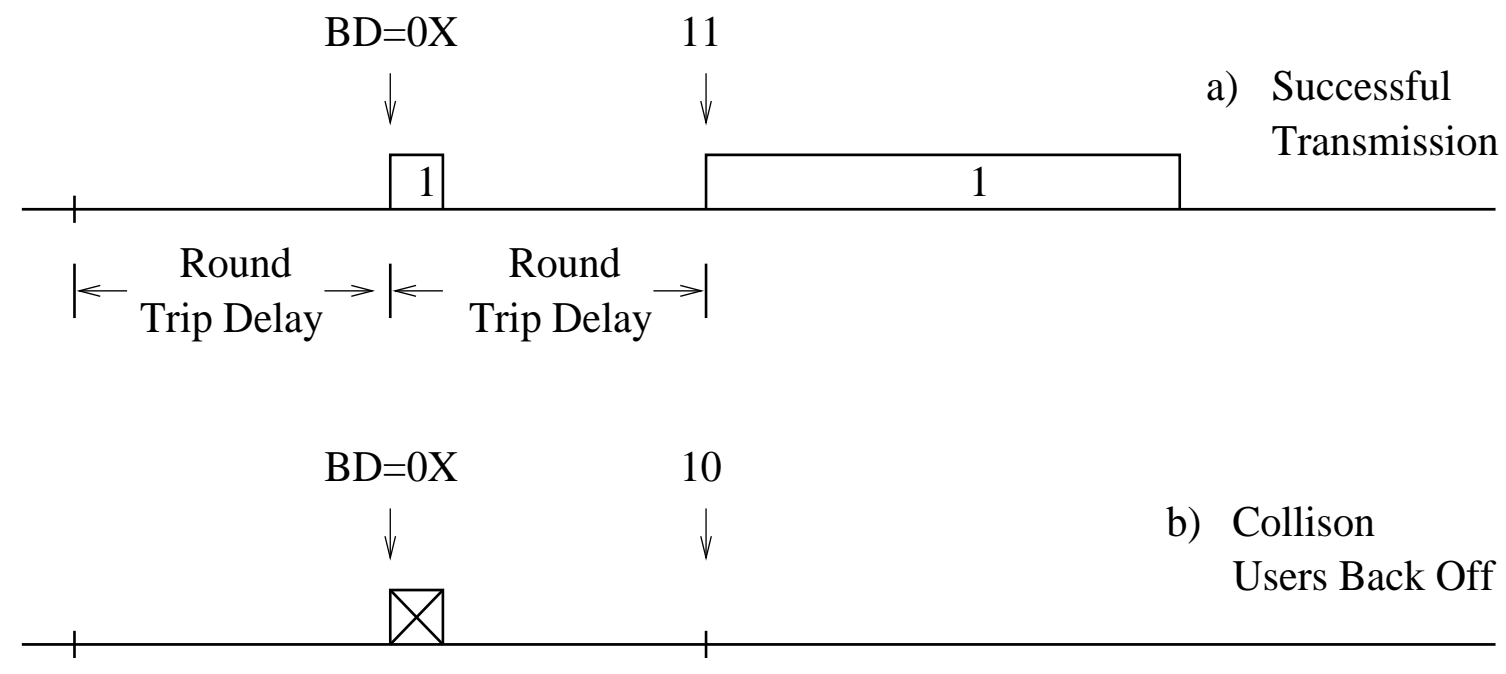

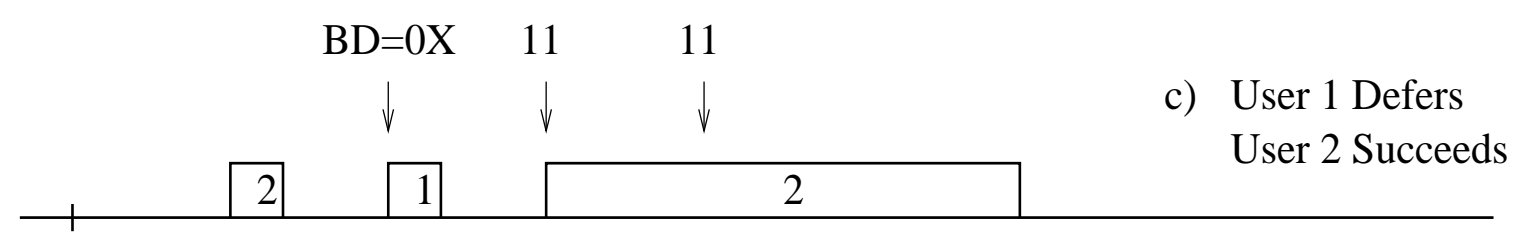

Figure 1. Timing Diagram for the DSMA/DT Protocol 


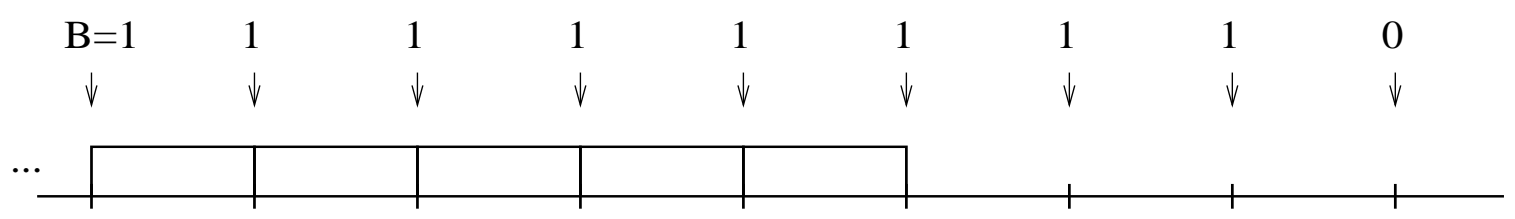

$\leftarrow$ Round Trip Delay $\rightarrow$

a) Regular Channel Busy/Idle Bit

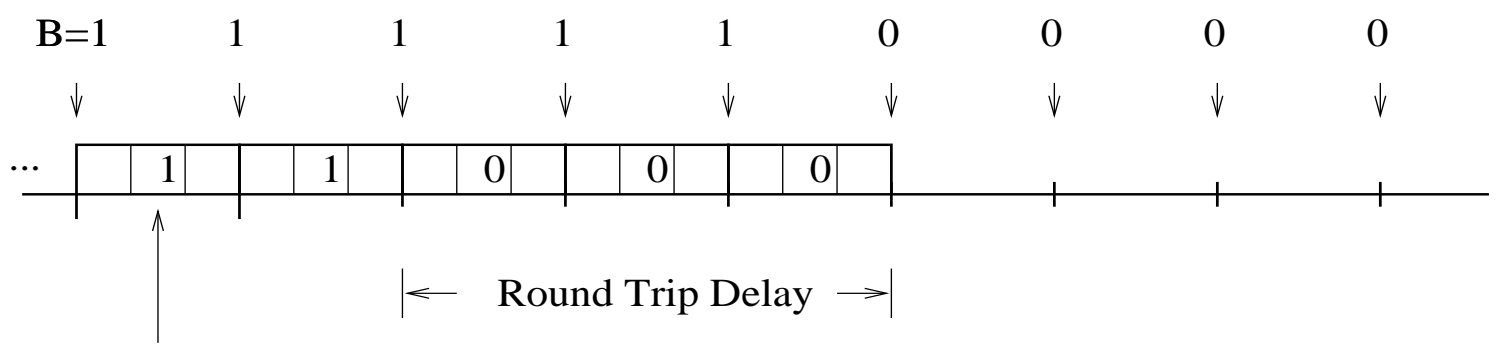

Length Indicator Bit

b) Look-Ahead Channel Busy/Idle Bit

Figure 2. Look-Ahead Busy/Idle Flag for the DSMA/DT Protocol 


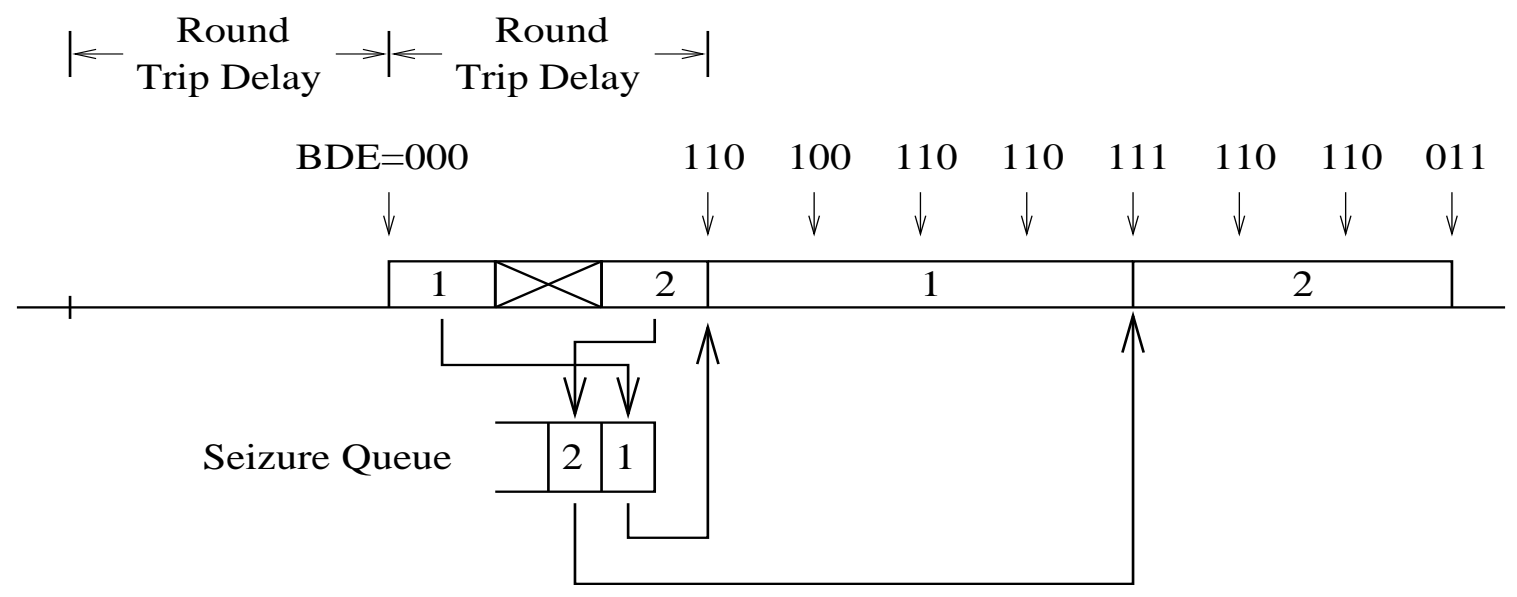

Figure 3. The DSMA/DT Protocol with Look-Ahead Flag and Seizure Queueing

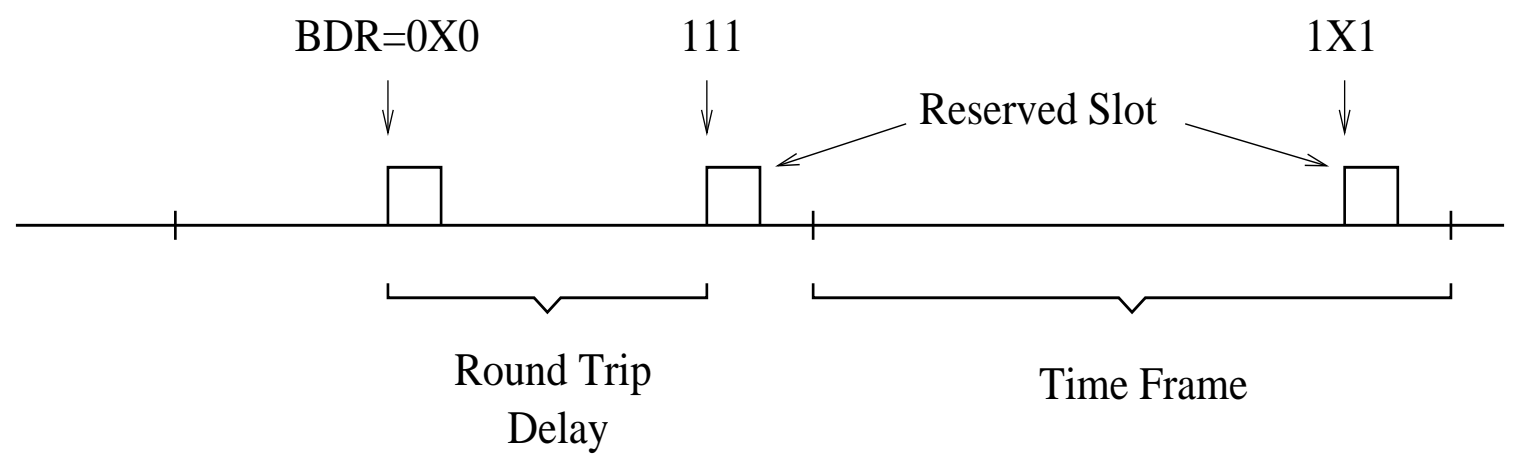

Figure 4. The DSMA/DT Protocol with Reserved Time Slots 


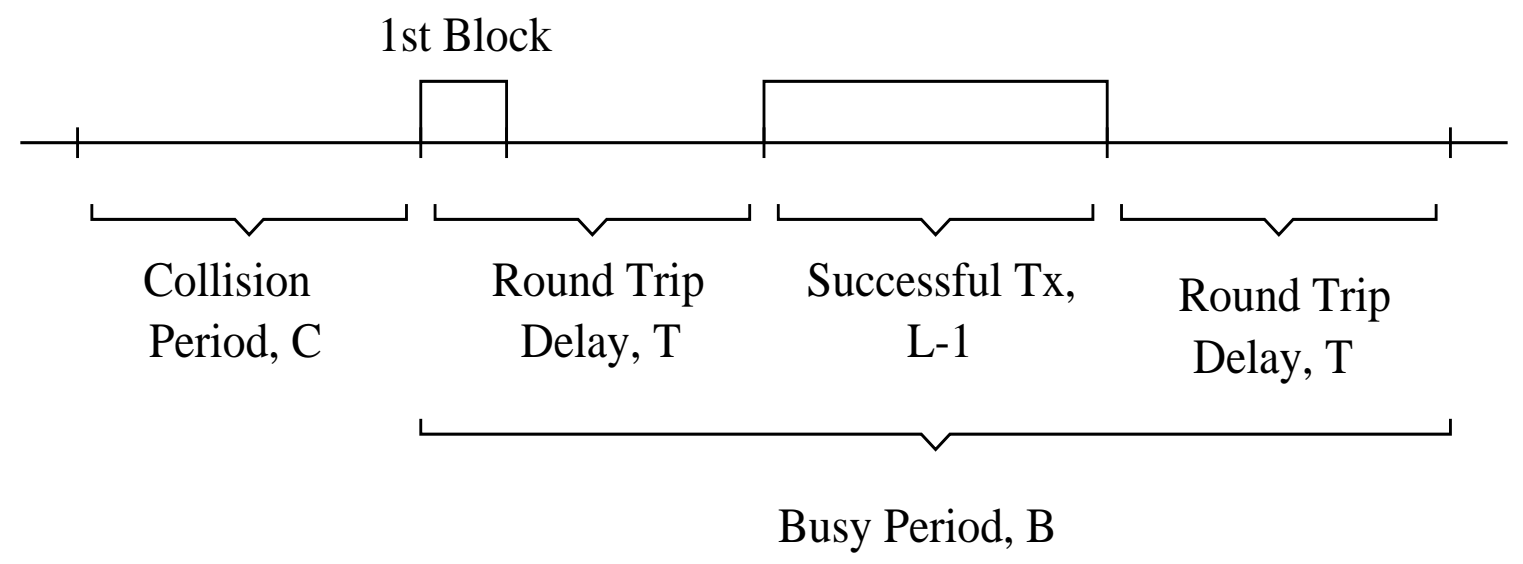

Figure 5. Timing Diagram for the DSMA/DT Throughput Analysis 
Figure 6. Throughput Comparison for Short Packet

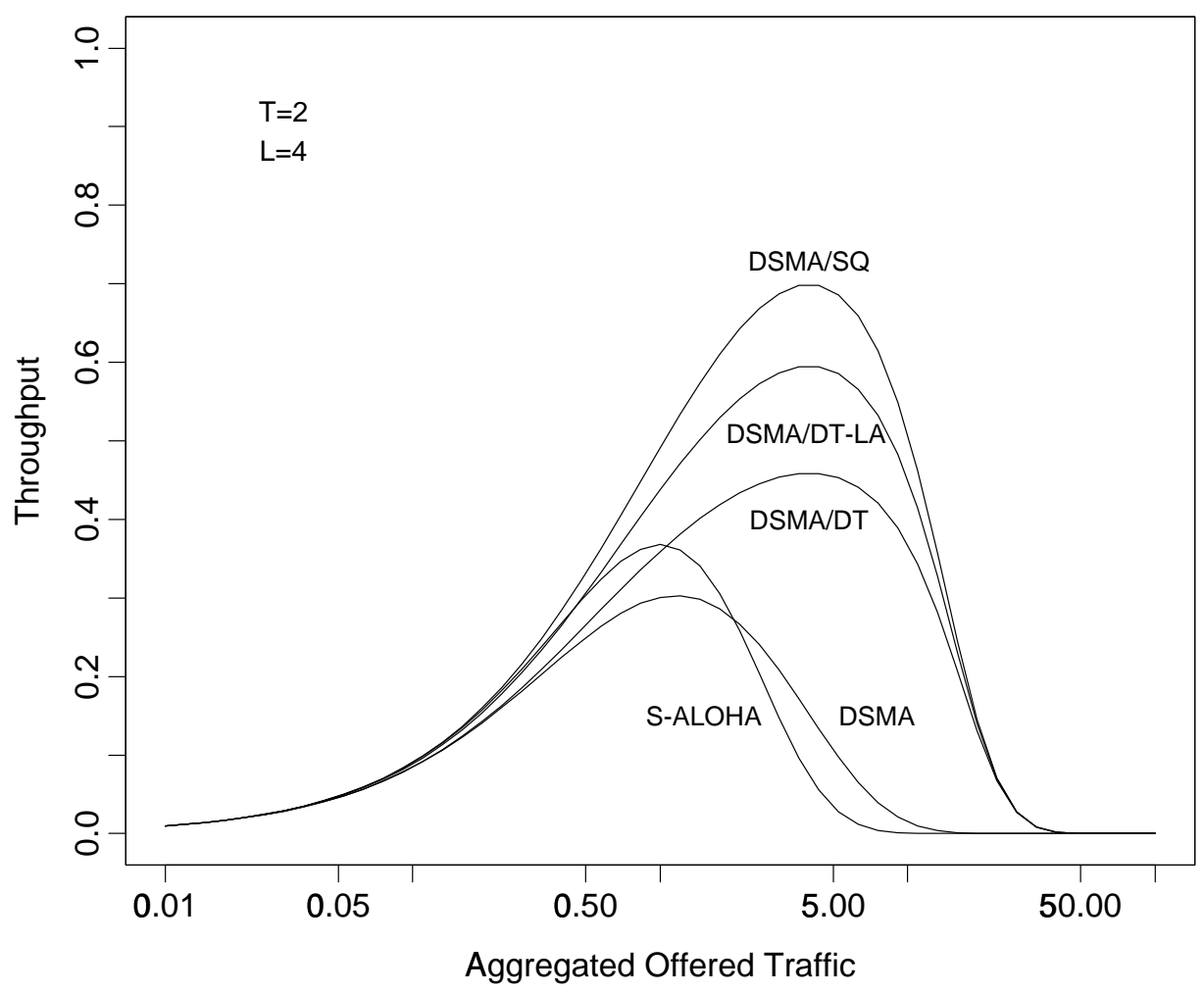

Figure 7. Throughput Comparison for Long Packet

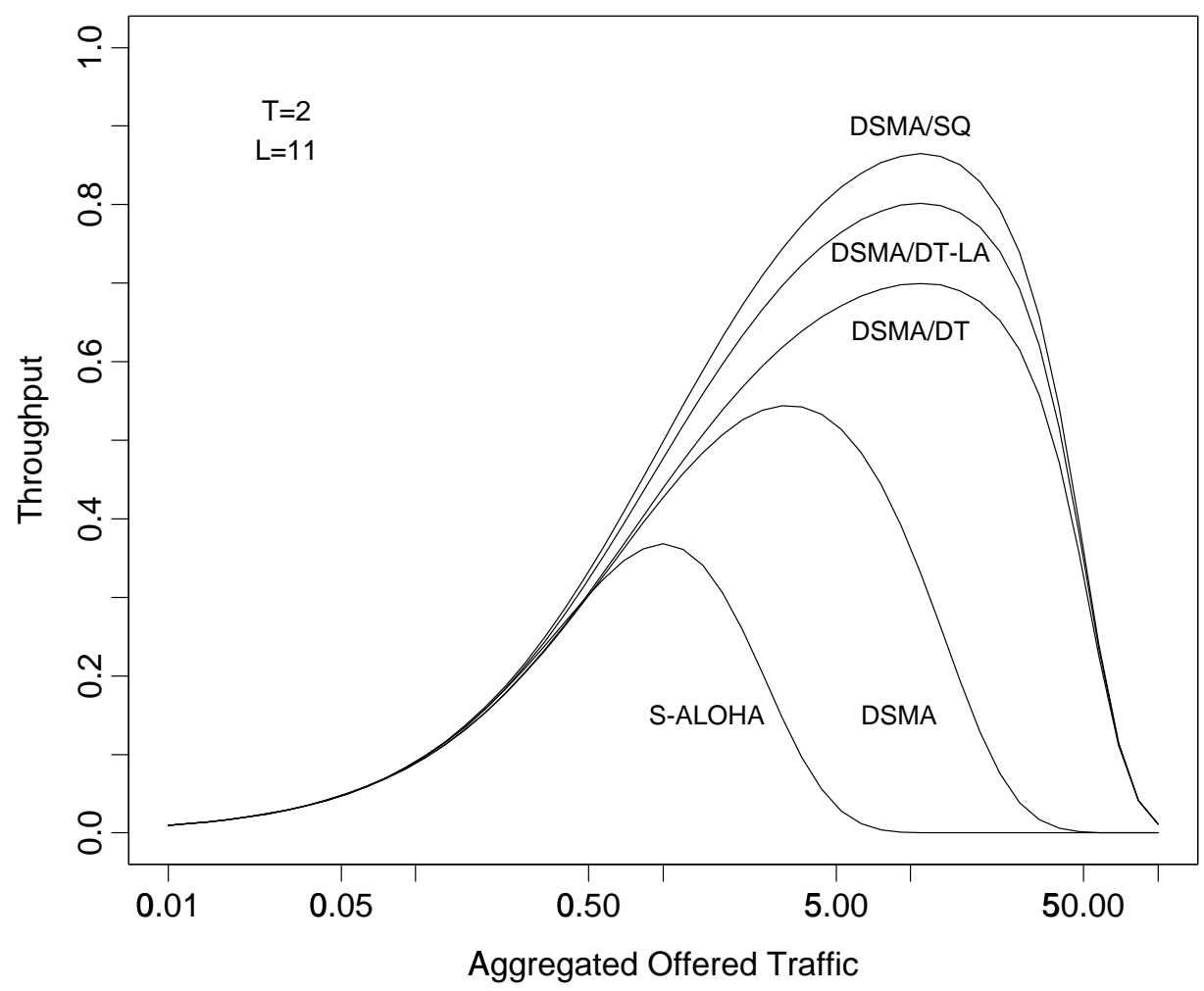

